\title{
Impact of pretreatment plasma D-dimer levels and its perioperative change on prognosis in operable esophageal squamous cell carcinoma
}

\author{
Jianbo $\mathrm{Li}^{1}$, Zhifan Zheng ${ }^{1}$ and Min Fang ${ }^{2}$ \\ ${ }^{1}$ Department of Radiotherapy, Ningbo Mingzhou Hospital, Ningbo, China \\ ${ }^{2}$ Department of Radiotherapy, Zhejiang Provincial People's Hospital, Hangzhou, China \\ Correspondence to: Jianbo Li, email: drlijianbo99@gmail.com
}

Keywords: esophageal squamous cell carcinoma, D-dimer, surgery, prognosis

Received: September 14, 2016 Accepted: June 02, 2017 Published: June 16, 2017

Copyright: Li et al. This is an open-access article distributed under the terms of the Creative Commons Attribution License 3.0 (CC BY 3.0 ), which permits unrestricted use, distribution, and reproduction in any medium, provided the original author and source are credited.

\section{ABSTRACT}

The aim of this study was to investigate the relationship between plasma D-dimer levels and its perioperative change and clinicopathological parameters in patients with operable esophageal squamous cell carcinoma (ESCC). We also analyzed their prognostic significance in ESCC patients. The data of 294 ESCC patients between December 2007 and December 2012 in Mingzhou hospital, Ningbo, China were analyzed retrospectively. Plasma D-dimer levels were measured one week before surgery and on the thirtieth postoperative day. The association between plasma D-dimer levels and clinicopathological parameters was evaluated. Kaplan-Meier survival analysis and Cox proportional hazards models were used to estimate the effect of plasma D-dimer levels and its perioperative change on disease-free survival (DFS) and overall survival (OS). Plasma D-dimer levels were above $0.5 \mu \mathrm{g} / \mathrm{mL}$ in 148 patients (50.3\%). Plasma D-dimer levels were significantly related with DFS $(P<0.001)$ and OS $(P<0.001)$ in univariate analysis. There was significant relationship between plasma D-dimer levels and DFS in patients with $N_{0}(P<0.001)$ or $N_{+}(P=0.003)$. Multivariate analysis revealed that plasma $D$-dimer levels $(P<0.001)$, sex $(P=0.012)$, and T stage $(P=0.033)$ were independent prognostic factors for DFS. Tumor length $(P=0.018)$, T stage $(P=0.008)$ and plasma $D$-dimer levels $(P=0.001)$ qualified as independent prognostic factors for OS. Our study suggests that pretreatment plasma D-dimer levels is a powerful independent prognostic factor for operable ESCC. Further studies are needed to prospectively validate this prognostic model and investigate the mechanisms underlying the correlation between elevated plasma D-dimer levels and poor prognosis in operable ESCC.

\section{INTRODUCTION}

Esophageal cancer is the sixth leading cause of cancer-related mortality in both developing and developed countries [1]. Esophageal squamous cell carcinoma (ESCC) is the most prevalent type of esophageal cancer, particularly in East Asia and some parts of Europe [2]. Despite recently much more effort has been dedicated to improving treatment of patients suffering from ESCC, the prognosis remains quite poor, with a great number of patients experiencing disease progression in a short time [3]. Radical surgery is standard treatment that can provide opportunity for cure. However, the 5 -year survival rates for surgically resectable ESCC are still unsatisfactory and range from $15 \%$ to $30 \%$ [4-6]. Adjuvant chemotherapy or radiotherapy could improve disease-free survival [7]. Furthermore, the benefit of platinum-based adjuvant chemotherapy for ESCC patients has not been established. Several studies have attempted to discover molecular biomarkers to predict the prognosis of ESCC [8-10]. However, to date, most of these markers had not been proven to be sufficiently effective [11]. 
D-dimer is a degradation product of fibrin in the blood during fibrinolysis, which could be used for diagnosis of thrombosis. Elevated plasma D-dimer levels were observed in acute venous thromboembolism, pregnancy, infectious diseases, as well as cancers $[12,13]$. Furthermore, D-dimer has been implicated in tumor invasion, metastasis, and eventual worse outcome in various types of cancer [14, 15]. In ESCC, plasma D-dimer levels are useful for predicting lymph node metastasis [16]. In Tomimaru Y et al.' report, plasma D-dimer levels correlated significantly with clinical and pathological responses to neoadjuvant chemotherapy [17]. However, the sample of these studies was relatively small. Furthermore, less studies have been performed to access the prognostic significance of postoperative plasma D-dimer levels after curative resection, which could reflect the patients' status after tumor removal. Therefore, the aim of this study was to determine whether the plasma D-dimer levels before treatment and its perioperative change are predictors of mortality in patients with ESCC.

\section{RESULTS}

\section{Patients}

The baseline characteristics of 294 patients are presented in Table 1. 268 patients were male and 26 patients were female. The median age was 58 years (range: 38-70 years). 245 patients were former smoker. After surgery, 195 patients were detected to have $\mathrm{T}_{3-4}$ disease while 99 patients had $\mathrm{T}_{1-2}$ disease. There were 96 patients with $\mathrm{N}_{0}$ and 198 patients with mediastinum lymph node metastasis. The median tumor length was $4 \mathrm{~cm}$ (range: $0.5 \mathrm{~cm}-11.0 \mathrm{~cm}$ ). Sixty patients $(20.4 \%)$ received four to six cycles of cisplatin-based chemotherapy while sixty-two $(21.1 \%)$ patients received adjuvant radiotherapy.

\section{Relationship between D-dimer and clinicopathological characteristics}

The relationship between several clinicopathological characteristics, such as sex, age, tumor location, smoking status, tumor length, T stage and $\mathrm{N}$ stage are summarized in Table 1. Plasma D-dimer levels were above $0.5 \mu \mathrm{g} / \mathrm{mL}$ in 148 patients $(50.3 \%)$. Tumor cell differentiation was related with plasma D-dimer levels $(P=0.025)$. Patients with low thoracic ESCC had higher plasma D-dimer levels, compared with upper or middle thoracic ESCC $(P=0.016)$. Besides, plasma D-dimer levels were also significant associated with T stage $(P<0.001)$ and $\mathrm{N}$ stage $(P=0.001)$. There were no correlation between plasma D-dimer levels and sex, age and smoking status $(P>0.05)$.

The clinicopathological features of two groups categorized by perioperative change of plasma D-dimer levels are summarized in Table 1. The patients' distribution was 254 patients in D-dimer decreased group and 40 patients in D-dimer increased group. There were no significant differences among the perioperative change of plasma D-dimer levels with regard to sex, age, smoking status, tumor location, tumor length or T stage, whereas $\mathrm{N}$ stage showed significant difference $(P<0.001)$.

\section{Effect of plasma D-dimer levels on DFS and OS by survival analysis}

During the follow-ups, 111 patients (37.8\%) experienced distant organ metastasis, while 38 patients (12.9\%) experienced local-regional recurrence. 97 patients $(33.0 \%)$ died of disease progression finally. The 3-year DFS and OS rates were $48.9 \%$ and $59.7 \%$, respectively. We performed univariate analysis for plasma D-dimer levels and other clinicopathological variables to find out the useful prognostic factors, which shown in Table 2. Sex $(P=0.031)$, T stage $(P<0.001), \mathrm{N}$ stage $(P=0.012)$, plasma D-dimer levels $(P<0.001)$ and perioperative change of plasma D-dimer levels $(P=0.021)$ were significantly related with DFS in univariate analysis. Tumor length $(P=0.007)$, $\mathrm{T}$ stage $(P<0.001), \mathrm{N}$ stage $(P=0.030)$, plasma D-dimer levels $(P<0.001)$ and perioperative change of plasma D-dimer levels $(P=0.026)$ were five significant prognostic factors related with OS. Patients with high plasma D-dimer levels had shorter DFS and OS than that with normal plasma D-dimer (3 year DFS rate: $36.8 \%$ Vs $62.9 \%, P<0.001$, Figure 1A; 3 year OS rate: $47.2 \% \mathrm{Vs} 72.3 \%, P<0.001$, Figure 1B). Similarly, DFS and OS were significantly shorter in patients with increased plasma D-dimer level than in those with decreased plasma D-dimer level (3 year DFS rate: $36.5 \%$ Vs $51.0 \%, P=0.021$, Figure $2 \mathrm{~A} ; 3$ year OS rate: $47.5 \%$ Vs $61.9 \%, P=0.026$, Figure $2 \mathrm{~B})$. In further analysis, high plasma D-dimer levels were significantly associated with shorter OS for $\mathrm{T}_{1-2}$ patients $(P=0.006)$ and for $\mathrm{T}_{3-4}$ patients $(P=0.005)$. Three year OS rate was $66.8 \%$ for high plasma D-dimer levels and $85.8 \%$ for normal plasma D-dimer levels in $\mathrm{T}_{1-2}$ patients, and $42.7 \%$ for high plasma $\mathrm{D}$-dimer levels and $61.5 \%$ for normal plasma $\mathrm{D}$-dimer levels in $\mathrm{T}_{3-4}$ patients. There was significant relationship between plasma D-dimer levels and DFS in patients with $\mathrm{N}_{0}(P<0.001)$ or $\mathrm{N}_{+}(P=0.003)$. Similarly, high plasma D-dimer levels were significantly associated with shorter $\mathrm{OS}$ for $\mathrm{N}_{0}$ patients $(P<0.001)$ and for $\mathrm{N}_{+}$patients $(P=0.005)$. High plasma D-dimer levels were significantly associated with shorter OS for patients with adjuvant chemotherapy $(P=0.045)$ and for patients without chemotherapy $(P=0.019)$. There was significant difference between plasma D-dimer levels and OS in patients with postoperative radiotherapy $(P=0.032)$ or without postoperative radiotherapy $(P=0.029)$.

Then, we performed multivariate analysis on the factors that were statistically significant in the univariate analysis. The results are shown in Table 3. The Cox proportional hazards regression indicated that plasma D-dimer levels $(P<0.001)$, sex $(P=0.012)$, and T stage 
Table 1: Relationship between clinicopathological parameters, plasma D-dimer level and INR in patients with ESCC

\begin{tabular}{|c|c|c|c|c|c|c|c|}
\hline \multirow[t]{2}{*}{ Variables } & \multirow[t]{2}{*}{ Patients $(n)$} & \multicolumn{2}{|c|}{ Plasma D-dimer level } & \multirow[t]{2}{*}{$P$} & \multicolumn{2}{|c|}{$\begin{array}{c}\text { Perioperative change of } \\
\text { D-dimer level }\end{array}$} & \multirow[t]{2}{*}{$P$} \\
\hline & & Normal $(\leq 0.5 \mu \mathrm{g} / \mathrm{ml})$ & High $(>0.5 \mu \mathrm{g} / \mathrm{ml})$ & & Decreased & Increased & \\
\hline \multicolumn{8}{|l|}{ Gender } \\
\hline Female & 26 & $11(42.3)$ & $15(57.7)$ & 0.539 & $25(96.2)$ & $1(3.8)$ & 0.128 \\
\hline Male & 268 & $135(50.4)$ & $133(49.6)$ & & $229(85.4)$ & $39(14.6)$ & \\
\hline \multicolumn{8}{|l|}{ Age (years) } \\
\hline$<65 \mathrm{Y}$ & 238 & $119(50.0)$ & $119(50.0)$ & 0.882 & $209(87.8)$ & $29(12.2)$ & 0.143 \\
\hline$\geq 65 \mathrm{Y}$ & 56 & $27(48.2)$ & $29(51.8)$ & & $45(80.4)$ & $11(19.6)$ & \\
\hline \multicolumn{8}{|l|}{ Smoking status } \\
\hline Never & 49 & $23(46.9)$ & $26(53.1)$ & 0.755 & $42(85.7)$ & $7(14.3)$ & 0.879 \\
\hline Ever & 245 & $123(50.2)$ & $122(50.2)$ & & $212(86.5)$ & $33(13.5)$ & \\
\hline \multicolumn{8}{|l|}{ Differentiation } \\
\hline Well & 32 & $24(75.0)$ & $8(25.0)$ & 0.025 & $30(93.8)$ & $2(6.3)$ & 0.168 \\
\hline Moderately & 206 & $97(47.1)$ & $109(52.9)$ & & $179(86.9)$ & $27(13.1)$ & \\
\hline Poorly & 51 & $23(45.1)$ & $28(54.9)$ & & $40(77.4)$ & $11(21.6)$ & \\
\hline Unknown & 5 & $2(40.0)$ & $3(60.0)$ & & $5(100)$ & $0(0)$ & \\
\hline \multicolumn{8}{|l|}{ Tumor location } \\
\hline Upper & 6 & $5(83.3)$ & $1(16.7)$ & 0.016 & $5(83.3)$ & $1(16.7)$ & 0.802 \\
\hline Middle & 139 & $78(56.1)$ & $61(43.9)$ & & $122(87.8)$ & $17(12.2)$ & \\
\hline Lower & 149 & $63(42.3)$ & $86(57.7)$ & & $127(85.2)$ & $22(14.8)$ & \\
\hline \multicolumn{8}{|l|}{ Tumor length } \\
\hline$\leq 5 \mathrm{~cm}$ & 219 & $115(52.5)$ & $104(47.5)$ & 0.060 & $194(87.8)$ & $27(12.2)$ & 0.227 \\
\hline$>5 \mathrm{~cm}$ & 73 & $29(39.7)$ & $44(60.3)$ & & $60(82.2)$ & $13(17.8)$ & \\
\hline \multicolumn{8}{|l|}{ T stage } \\
\hline $\mathrm{T}_{1-2}$ & 99 & $71(71.7)$ & $28(28.3)$ & $<0.001$ & $85(85.9)$ & $14(14.1)$ & 0.849 \\
\hline $\mathrm{T}_{3-4}$ & 195 & $75(38.5)$ & $120(61.5)$ & & $169(86.1)$ & $26(13.3)$ & \\
\hline \multicolumn{8}{|l|}{$\mathrm{N}$ stage } \\
\hline $\mathrm{N}_{0}$ & 96 & $112(56.6)$ & $86(43.4)$ & 0.001 & $56(58.3)$ & $40(41.7)$ & $<0.001$ \\
\hline $\mathrm{N}_{1-3}$ & 198 & $34(35.4)$ & $62(64.6)$ & & $188(94.9)$ & $10(5.1)$ & \\
\hline
\end{tabular}

$(P=0.033)$ were independent prognostic factors for DFS. Tumor length $(P=0.018)$, T stage $(P=0.008)$ and plasma D-dimer levels $(P=0.001)$ qualified as independent prognostic factors for OS. Patients with high plasma D-dimer levels had an elevated risk of disease progression and death compared to those with normal plasma D-dimer levels. The hazard ratio was 2.11 (95\% confidence interval [CI] 1.46-3.05) for disease progression and 2.25 (95\% CI 1.42-3.56) for death.

\section{DISCUSSION}

Hypercoagulability in patients with malignant tumors of gastrointestinal tract system is common and well known. In a large scale of 1042 gastric patients, plasma D-dimer levels were significantly elevated in metastasis GC patients, especially in patients with hematogenous visceral metastasis, and plasma D-dimer levels correlated with vascular cancer emboli in resected tissue samples [18]. In this present study, we demonstrated that plasma D-dimer levels were significant related with T stage $(P<0.001)$ and $\mathrm{N}$ stage $(P=0.001)$. Patients with low thoracic ESCC had higher plasma D-dimer levels, compared with upper or middle thoracic ESCC $(P=0.016)$. We also showed that high plasma D-dimer levels were significantly associated with decreased DFS and OS probability. Patients with elevated plasma D-dimer levels had 2.11 times the risk for disease progression and 2.25 times the risk for death compared with those with normal plasma D-dimer levels.

Diao et al. [19] examined plasma D-dimer levels between 66 patients with esophageal cancer and 12 patients with benign disease before and after operation. Plasma D-dimer levels were increased significantly higher in cancer group. OS time was significantly short in cancer patients whose plasma D-dimer levels on the 3rd and 9th post-operative day above the median number. In another report [16], plasma D-dimer levels were associated with the 
Table 2: Univariate analysis for clinicopathological parameters, plasma D-dimer levels, and perioperative change of D-dimer level associated with DFS and OS in patients with ESCC

\begin{tabular}{|c|c|c|c|c|}
\hline Variables & 3-year DFS rate & $P$ & 3-year OS rate & $P$ \\
\hline \multicolumn{5}{|l|}{ Sex } \\
\hline Female & 68.1 & 0.031 & 73.1 & 0.153 \\
\hline Male & 47.0 & & 57.9 & \\
\hline \multicolumn{5}{|l|}{ Age (years) } \\
\hline$<65 \mathrm{Y}$ & 48.6 & 0.532 & 62.0 & 0.455 \\
\hline$\geq 65 \mathrm{Y}$ & 50.3 & & 51.8 & \\
\hline \multicolumn{5}{|l|}{ Smoking status } \\
\hline Never & 54.9 & 0.266 & 66.3 & 0.242 \\
\hline Ever & 47.7 & & 58.5 & \\
\hline \multicolumn{5}{|l|}{ Differentiation } \\
\hline Well & 56.5 & 0.699 & 65.5 & 0.392 \\
\hline Moderately & 49.8 & & 59.8 & \\
\hline Poorly & 38.7 & & 52.6 & \\
\hline Unknown & 40.0 & & 50.0 & \\
\hline \multicolumn{5}{|l|}{ Tumor location } \\
\hline Upper + Middle & 55.0 & 0.095 & 66.8 & 0.102 \\
\hline Lower & 42.6 & & 52.4 & \\
\hline \multicolumn{5}{|l|}{ Tumor length } \\
\hline$\leq 5 \mathrm{~cm}$ & 50.7 & 0.126 & 62.3 & 0.007 \\
\hline$>5 \mathrm{~cm}$ & 41.4 & & 51.1 & \\
\hline \multicolumn{5}{|l|}{ T stage } \\
\hline $\mathrm{T}_{1-2}$ & 67.2 & $<0.001$ & 78.0 & $<0.001$ \\
\hline $\mathrm{T}_{3-4}$ & 39.6 & & 49.3 & \\
\hline \multicolumn{5}{|l|}{$\mathrm{N}$ stage } \\
\hline $\mathrm{N}_{0}$ & 55.0 & 0.012 & 65.2 & 0.030 \\
\hline $\mathrm{N}_{1-3}$ & 37.8 & & 49.3 & \\
\hline \multicolumn{5}{|l|}{ Adjuvant chemotherapy } \\
\hline Yes & 36.9 & 0.175 & 54.9 & 0.627 \\
\hline No & 52.6 & & 61.1 & \\
\hline \multicolumn{5}{|l|}{ Adjuvant radiotherapy } \\
\hline Yes & 41.2 & 0.286 & 54.1 & 0.090 \\
\hline No & 51.2 & & 61.3 & \\
\hline \multicolumn{5}{|l|}{ Plasma D-dimer level } \\
\hline Normal $(\leq 0.5 \mu \mathrm{g} / \mathrm{ml})$ & 62.9 & $<0.001$ & 73.9 & $<0.001$ \\
\hline High $(>0.5 \mu \mathrm{g} / \mathrm{ml})$ & 36.8 & & 47.2 & \\
\hline \multicolumn{5}{|l|}{$\begin{array}{l}\text { Perioperative change of } \\
\text { D-dimer level }\end{array}$} \\
\hline Decreased & 51.0 & 0.021 & 61.9 & 0.026 \\
\hline Increased & 36.5 & & 47.5 & \\
\hline
\end{tabular}

Abbreviations: DFS, Disease-free survival; OS, Overall survival; ESCC, Esophageal squamous cell carcinoma. 


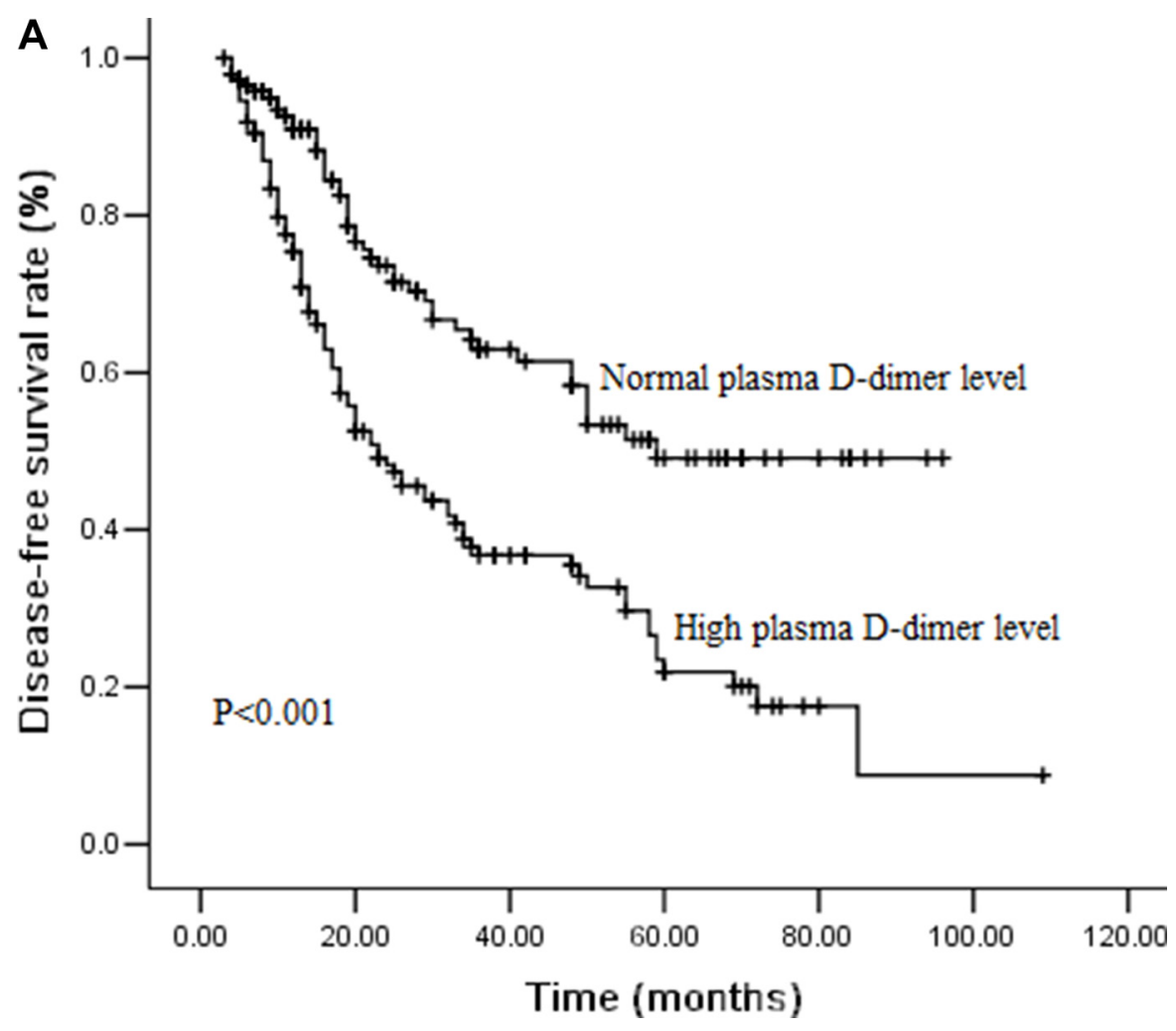

Figure 1A: Cumulative survival curves for disease-free survival (DFS) time according to pretreatment plasma D-dimer levels.

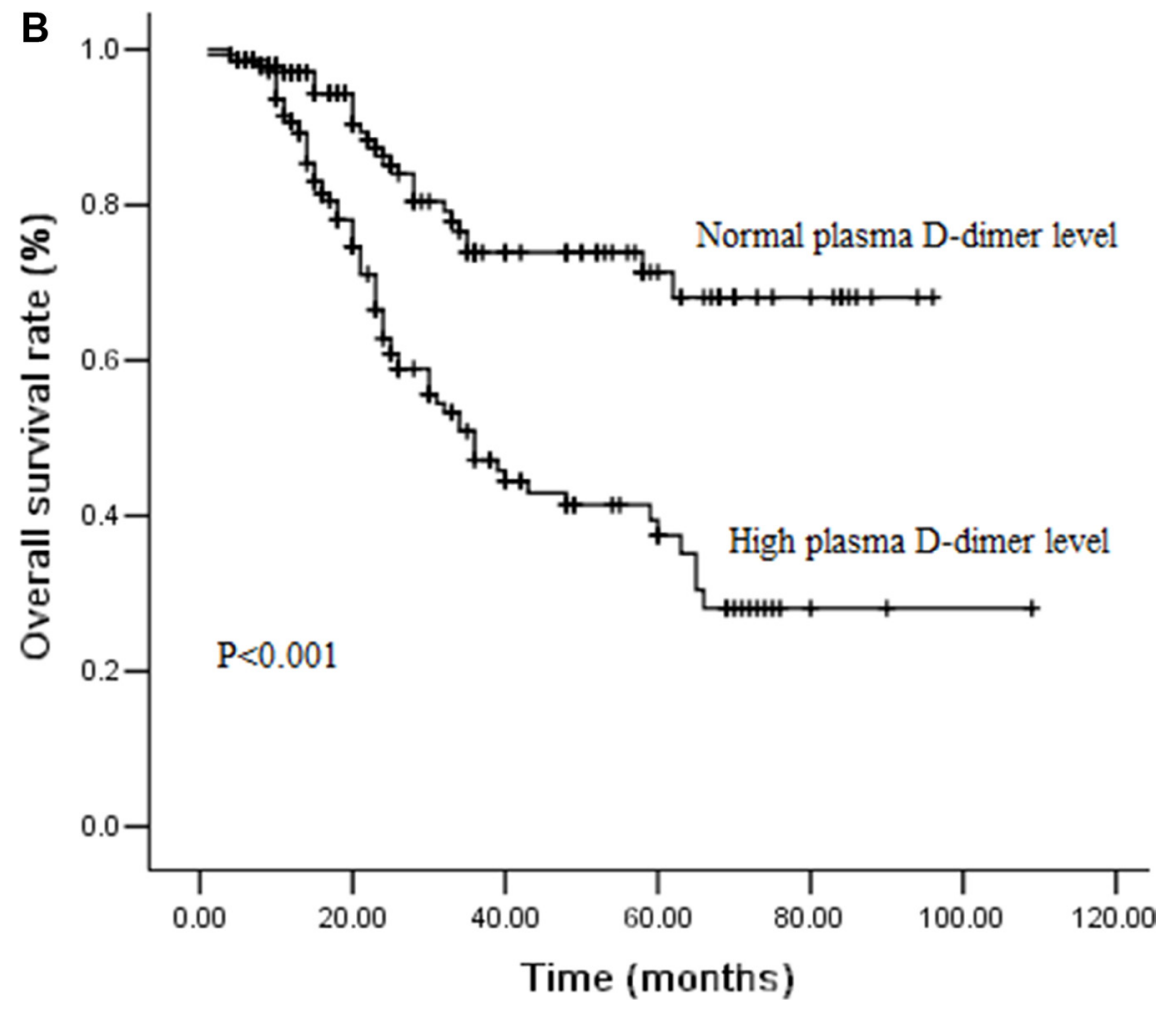

Figure 1B: Cumulative survival curves for overall survival (OS) time according to pretreatment plasma D-dimer levels. 


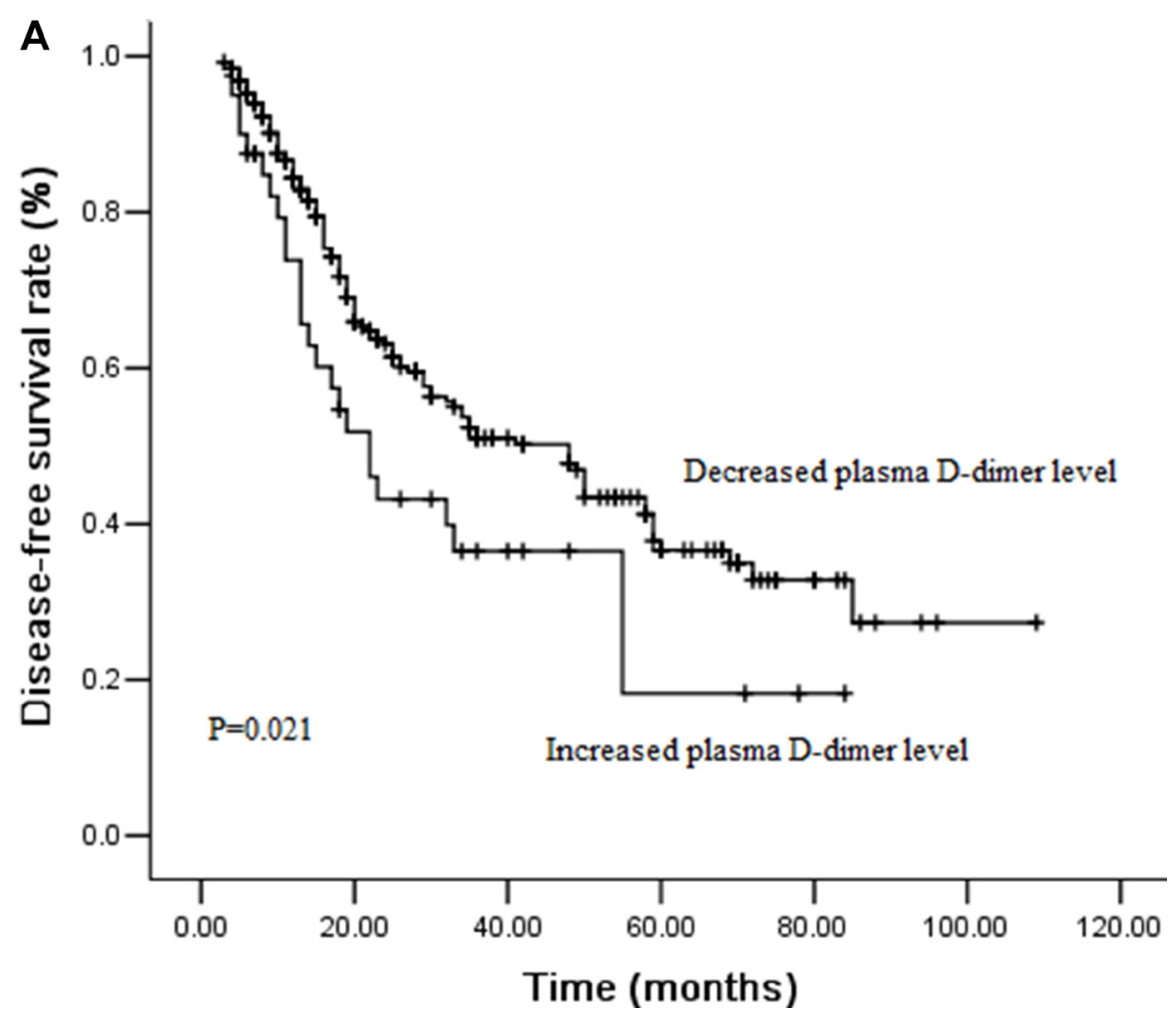

Figure 2A: Cumulative survival curves for disease-free survival (DFS) according to perioperative change of plasma D-dimer levels.

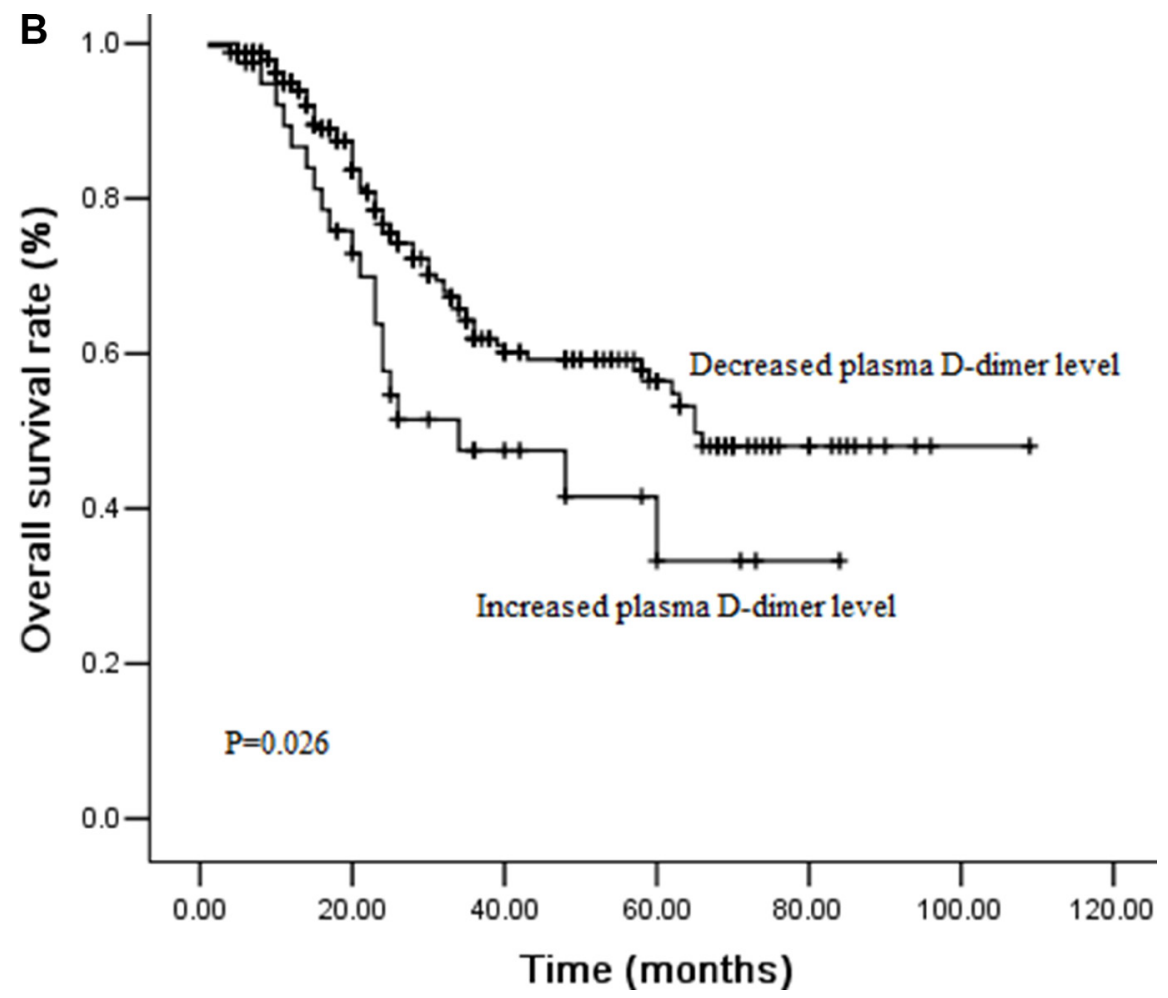

Figure 2B: Cumulative survival curves for overall survival (OS) time according to perioperative change of plasma D-dimer levels. 
Table 3: Results of multivariate analyses of clinicopathological factors affecting DFS and OS in patients with ESCC

\begin{tabular}{lcccccc}
\hline \multicolumn{1}{c}{ Variables } & \multicolumn{3}{c}{ DFS } & \multicolumn{3}{c}{ OS } \\
\cline { 2 - 7 } & RR & $\mathbf{9 5 \%}$ CI & $\boldsymbol{P}$ & RR & $\mathbf{9 5 \% ~ C I ~}$ & $\boldsymbol{P}$ \\
\hline Sex (male Vs female) & 2.47 & $1.19-5.13$ & 0.016 & - & - & - \\
Tumor length $(>5 \mathrm{~cm}$ Vs $\leq 5 \mathrm{~cm})$ & - & - & - & 1.66 & $1.07-2.57$ & 0.023 \\
T stage $\left(\mathrm{T}_{3-4} \mathrm{Vs}_{1-2}\right)$ & 1.59 & $1.04-2.42$ & 0.033 & 2.20 & $1.25-3.85$ & 0.006 \\
$\mathrm{~N}$ stage $\left(\mathrm{N}_{1-3} \mathrm{Vs} \mathrm{N}_{0}\right.$ ) & 1.13 & $0.73-1.75$ & 0.571 & 1.03 & $0.61-1.74$ & 0.926 \\
Plasma D-dimer level (High Vs normal) & 2.09 & $1.43-3.05$ & $<0.001$ & 2.23 & $1.39-3.57$ & 0.001 \\
Perioperative change of D-dimer level (Increased & 1.07 & $0.61-1.86$ & 0.813 & 1.28 & $0.67-2.43$ & 0.451 \\
Vs Decreased) & & & & & & \\
\hline
\end{tabular}

Abbreviations: DFS, Disease-free survival; OS, Overall survival; ESCC, Esophageal squamous cell carcinoma; RR, relative risk; CI, confidence interval.

number of lymph node metastasis $(P<0.001)$. Compared with other serum tumor markers, such as carcinoembryonic antigen (CEA) and squamous cell carcinoma-related antigen (SCC-Ag), D-dimer is the best marker to predict $\mathrm{N}$ stage before operation. However, the sample of this study was relatively small and the author did not clarify the correlation between hematogenous metastasis and D-dimer. Tomimaru $\mathrm{Y}$ et al. [17] investigated the association between plasma D-dimer levels and chemotherapy response. Plasma D-dimer levels were significantly lower in patients response to chemotherapy. Although these results somewhat overlapped with our study, the strength of the current study was direct comparison of plasma D-dimer levels ESCC under the same treatment. We also performed sub-group analysis to investigate the role of plasma D-dimer levels in ESCC. Furthermore, patients were categorized into two groups according to their plasma D-dimer levels' variation before and after operation: Increased = plasma D-dimer levels increasing in the perioperative period; Decreased = plasma D-dimer levels decreasing in the perioperative period. Effect of perioperative change of plasma D-dimer levels on DFS and OS was evaluated by survival analysis.

Our study also had several limitations. First, plasma D-dimer levels and platelet counts were checked only once in each patient and were not examined during the treatment and the follow-up. The relationship between changes in plasma $\mathrm{D}$-dimer levels and tumor progression need to be investigated. Second, our study included a comparative homogeneous population with the majority of male and smoker patients, which might cause a bias. Also, this is a retrospective study based on patients of one institution and could not completely avoid selection bias. Furthermore, distant organ metastasis rate during follow-up was not the focus in this study, they should be reported. But unfortunately, we do not have detailed information of metastasis due to the retrospective nature of this study.

\section{MATERIALS AND METHODS}

\section{Patients}

In this study, the data of 294 ESCC patients between December 2007 and December 2012 in Mingzhou hospital, Ningbo, China were analyzed retrospectively. All patients were newly confirmed to have ESCC and had not received treatment previously. Patients with other malignancies were excluded from this study. Pretreatment plasma D-dimer levels and serum CRP levels obtained within 1 week before surgery. Patients with a history of venous thrombosis or anticoagulation therapy, hypertension, cardiovascular and cerebrovascular disease and diabetes were excluded from the current study. The following detail clinical information was retrospectively collected and analyzed for each case: sex, age at treatment, smoking status, tumor location, clinical TNM stage, treatment response, disease-free survival (DFS) and overall survival (OS) after treatment. TNM classification was evaluated according to the 7th edition of the Union for International Cancer Control staging. All procedures performed in studies involving human participants were in accordance with the ethical standards of the institutional and/or national research committee. Informed consent was obtained from all individual participants included in the study.

\section{D-dimer measurement}

As a part of clinical routine examinations, plasma D-dimer levels were measured 1 week before surgery and on the thirtieth postoperative day. Each patient provided two 5 $\mathrm{mL}$ blood samples before breakfast. Plasma D-dimer levels were measured by a latex-enhanced immunoturbidimetric assay using a Sysmex CA 7000 (Sysmex Corp, Kobe, Japan) analyzer according to manufacturer's instruction in Mingzhou hospital. Plasma D-dimer levels of $0.5 \mu \mathrm{g} /$ $\mathrm{mL}$ was used as cutoff for normal versus high $\mathrm{D}$-dimer 
values, according to the manufacture's recommendation. Furthermore, patients were categorized into two groups according to their plasma D-dimer levels' variation before and after operation: Increased = plasma D-dimer levels increasing in the perioperative period; Decreased = plasma D-dimer levels decreasing in the perioperative period.

\section{Treatment}

All patients underwent total or subtotal transthoracic esophagectomy and regional lymphadenectomy with curative intent. Sixty (20.4\%) were treated with adjuvant platinum-based chemotherapy and sixty-two (21.1\%) patients received post-operative radiotherapy. All patients received standardized follow-up at a 3-month interval for the first 2 years after operation, a 6-month interval in the third year and yearly thereafter. The median follow-up time was 26 months.

\section{Statistical analysis}

Disease-free survival (DFS) was defined as the time from surgery to any recurrence, including locoregional recurrence and distant organ metastasis. Overall survival (OS) was calculated as the time from the date of surgery to death or censoring. The chi-square test was performed to evaluate the association between the clinicopathological variables and plasma D-dimer levels and INR. Survival curves were estimated by the univariate Kaplan-Meier method. The log-rank test was applied to check the significant difference in the curves among groups. Furthermore, we used the Cox proportional hazards model for multivariate analysis. All statistical calculations were performed with SPSS 23.0 software for Windows (Chicago, IL, USA). Two-sided $P$-values of $<0.05$ were considered to be statistically significant.

\section{CONCLUSIONS}

In conclusion, we have provided the clinical evidence that plasma D-dimer levels were related with clinicopathological factors in ESCC. We demonstrated that pretreatment plasma D-dimer levels could be served as new independent prognostic biomarkers for DFS and OS in operable ESCC, although its prognostic significance still requires confirmation with larger patient populations.

\section{Authors' contributions}

All authors contributed toward data analysis, drafting and critically revising the paper and agree to be accountable for all aspects of the work.

\section{CONFLICTS OF INTEREST}

The authors declare that they have no competing interests.

\section{REFERENCES}

1. Miller KD, Siegel RL, Lin CC, Mariotto AB, Kramer JL, Rowland JH, Stein KD, Alteri R, Jemal A. Cancer treatment and survivorship statistics, 2016. CA Cancer J Clin. 2016; 66:271-289.

2. Pickens A, Orringer MB. Geographical distribution and racial disparity in esophageal cancer. Ann Thorac Surg. 2003; 76:S1367-1369.

3. Lee PC, Mirza FM, Port JL, Stiles BM, Paul S, Christos P, Altorki NK. Predictors of recurrence and disease-free survival in patients with completely resected esophageal carcinoma. J Thorac Cardiovasc Surg. 2011; 141:1196-1206.

4. Le Bras GF, Farooq MH, Falk GW, Andl CD. Esophageal cancer: the latest on chemoprevention and state of the art therapies. Pharmacol Res. 2016; 113:236-244.

5. Xiao ZF, Yang ZY, Liang J, Miao YJ, Wang M, Yin WB, Gu XZ, Zhang DC, Zhang RG, Wang LJ. Value of radiotherapy after radical surgery for esophageal carcinoma: a report of 495 patients. Ann Thorac Surg. 2003; 75:331-336.

6. Teniere P, Hay JM, Fingerhut A, Fagniez PL. Postoperative radiation therapy does not increase survival after curative resection for squamous cell carcinoma of the middle and lower esophagus as shown by a multicenter controlled trial. French University Association for Surgical Research. Surg Gynecol Obstet. 1991; 173:123-130.

7. Ando N, Iizuka T, Ide H, Ishida K, Shinoda M, Nishimaki T, Takiyama W, Watanabe H, Isono K, Aoyama N, Makuuchi H, Tanaka O, Yamana H, et al. Surgery plus chemotherapy compared with surgery alone for localized squamous cell carcinoma of the thoracic esophagus: a Japan Clinical Oncology Group Study_JCOG9204. J Clin Oncol. 2003; 21:4592-4596.

8. Wang S, Wang Z, Yang YU, Shi MO, Sun Z. Overexpression of Ku80 correlates with aggressive clinicopathological features and adverse prognosis in esophageal squamous cell carcinoma. Oncol Lett. 2015; 10:2705-2712.

9. Okumura H, Uchikado Y, Omoto I, Motomura M, Kita Y, Sasaki K, Noda M, Arigami T, Uenosono Y, Baba K, Mori S, Kijima Y, Nakajo A, et al. Ferredoxin Reductase Is Useful for Predicting the Effect of Chemoradiation Therapy on Esophageal Squamous Cell Carcinoma. Anticancer Res. 2015; 35:6471-6474.

10. Liu F, Gu L, Cao Y, Fan X, Zhang F, Sang M. Aberrant overexpression of EZH2 and $\mathrm{H} 3 \mathrm{~K} 27 \mathrm{me} 3$ serves as poor prognostic biomarker for esophageal squamous cell carcinoma patients. Biomarkers. 2016; 21:80-90.

11. Hong L, Han Y, Zhang H, Fan D. Prognostic markers in esophageal cancer: from basic research to clinical use. Expert Rev Gastroenterol Hepatol. 2015; 9:887-889.

12. Inal $\mathrm{T}$, Anar $\mathrm{C}$, Polat $\mathrm{G}$, Unsal I, Halilcolar H. The prognostic value of D-dimer in lung cancer. Clin Respir J. 2015; 9:305-313. 
13. Komurcuoglu B, Ulusoy S, Gayaf M, Guler A, Ozden E. Prognostic value of plasma D-dimer levels in lung carcinoma. Tumori. 2011; 97:743-748.

14. Dirix LY, Salgado R, Weytjens R, Colpaert C, Benoy I, Huget P, van Dam P, Prove A, Lemmens J, Vermeulen P. Plasma fibrin D-dimer levels correlate with tumour volume, progression rate and survival in patients with metastatic breast cancer. Br J Cancer. 2002; 86:389-395.

15. Liu L, Zhang X, Yan B, Gu Q, Zhang X, Jiao J, Sun D, Wang N, Yue X. Elevated plasma D-dimer levels correlate with long term survival of gastric cancer patients. PLoS One. 2014; 9:e90547.

16. Tomimaru Y, Yano M, Takachi K, Kishi K, Miyashiro I, Ohue M, Ohigashi H, Sasaki Y, Ishikawa O, Imaoka S. Plasma D-dimer levels show correlation with number of lymph node metastases in patients with esophageal cancer. J Am Coll Surg. 2006; 202:139-145.
17. Tomimaru Y, Yano M, Takachi K, Kishi K, Miyashiro I, Ohue M, Ohigashi H, Sasaki Y, Ishikawa O, Imaoka S. Correlation between pretherapeutic d-dimer levels and response to neoadjuvant chemotherapy in patients with advanced esophageal cancer. Dis Esophagus. 2008; 21:281-287.

18. Diao D, Wang Z, Cheng Y, Zhang H, Guo Q, Song Y, Zhu K, Li K, Liu D, Dang C. D-dimer: not just an indicator of venous thrombosis but a predictor of asymptomatic hematogenous metastasis in gastric cancer patients. PLoS One. 2014; 9:e101125.

19. Diao D, Zhu K, Wang Z, Cheng Y, Li K, Pei L, Dang C. Prognostic value of the D-dimer test in oesophageal cancer during the perioperative period. J Surg Oncol. 2013; 108:34-41. 